\title{
Cateterismo de senos petrosos en el diagnóstico etiológico de síndrome de Cushing: primera experiencia en Uruguay
}

Petrosal sinus sampling for etiological diagnosis of Cushing's syndrome: first experience in Uruguay

Cateterismo dos seios petrosos no diagnóstico etiológico da síndrome de Cushing: primeira experiência no Uruguai

Mariana Pintadoํ․ Asistente Paula Montiglia¹, Prof. Agdo. Marcelo Langleib², Prof. Adj. Fernanda Sánchez³ , Lic. Flavia Varela³, Prof. Agdo. Ramiro Lima4, Margarita Vaz¹, Prof. Agda. María Mercedes Piñeyro ${ }^{1}$

\section{Resumen}

Identificar la causa de un síndrome de Cushing dependiente de adrenocorticotropina (ACTH) es esencial para realizar un tratamiento correcto. La hipersecreción de ACTH es debida en su mayoría a un tumor hipofisario (enfermedad de Cushing) o, en un 10\%-20\%, a tumores con producción ectópica de esta hormona. Los test no invasivos tienen baja sensibilidad y especificidad para diferenciar estas dos etiologías. El patrón oro lo constituye el cateterismo bilateral de los senos petrosos inferiores (CSP). Mediante el CSP se demuestra la hipersecreción de ACTH a nivel hipofisario al documentar un gradiente de ACTH central a periférico en el drenaje del tumor. Se recomienda realizarlo en todo síndrome de Cushing ACTH dependiente, aunque suele reservarse para pacientes con diagnóstico de hipercortisolismo y hallazgos negativos o equívocos en la resonancia nuclear magnética (RNM) de la región selar.

Presentamos el primer caso en Uruguay en que se utilizó el CSP como método diagnóstico, una mujer de 55 años que presentó un hipercortisolismo ACTH-dependiente con una imagen adenohipofisaria < $6 \mathrm{~mm}$. El gradiente petroso-periférico confirmó el diagnóstico de enfermedad de Cushing y no hubo complicaciones durante el procedimiento. Posteriormente se realizó la resección del adenoma mediante cirugía transesfenoidal, con buena evolución y confirmación inmunohistoquímica del tumor.

Palabras clave: Síndrome de Cushing Muestreo de seno petroso

Desmopresina

Cateterismo
Key words: $\quad$ Cushing syndrome

Petrosal sinus sampling

Desmopressin

Catheterization

1. Clínica de Endocrinología y Metabolismo, Hospital de Clínicas, Facultad de Medicina, Universidad de la República, Montevideo, Uruguay.

2. Departamento Clínico de Imagenología, Hospital de Clínicas, Facultad de Medicina, Universidad de la República, Montevideo, Uruguay.

3. Departamento de Laboratorio Clínico, Hospital de Clínicas, Facultad de Medicina, Universidad de la República, Montevideo, Uruguay.

4. Neurocirugía, Hospital de Clínicas, Facultad de Medicina, Universidad de la República, Montevideo, Uruguay.

Clínica de Endocrinología y Metabolismo, Facultad de Medicina, UdelaR, Montevideo, Uruguay.

Correspondencia: Maria M. Piñeyro, Avenida Italia s/n, Montevideo, Uruguay. Correo electrónico: mercepin@gmail.com

Los autores declaran no tener conflictos de intereses.

Fuente de financiación: ninguna.

Cuenta con consentimiento informado para su publicación

Recibido: 11/11/2020

Aprobado: $28 / 4 / 2021$ 


\section{Introducción}

El síndrome de Cushing (SC) es el conjunto de síntomas y signos que reflejan la exposición prolongada a niveles inapropiadamente elevados de glucocorticoides en los tejidos. Puede ser de origen exógeno o endógeno. Dentro de las causas endógenas, la más frecuente es la dependiente de la ACTH, hasta en un $80 \%-85 \%$ de los ca$\operatorname{sos}^{(1)}$. La hipersecreción de ACTH es debida en su gran mayoría $(80 \%-85 \%)$ a un tumor hipofisario (enfermedad de Cushing, EC), y en el resto a tumores con producción ectópica de esta hormona. Identificar la causa de un SC ACTH dependiente es esencial para realizar un tratamiento correcto. Dado que la presentación clínica y de laboratorio son similares en ambas causas, es difícil realizar un diagnóstico definitivo. Los test no invasivos tienen baja sensibilidad y especificidad para diferenciarlos. Además, muchos de los tumores ectópicos (sobre todo los carcinoides bronquiales) pueden permanecer ocultos por años a las técnicas de imágenes. El patrón oro para el diagnóstico diferencial entre $\mathrm{EC}$ y un tumor ectópico secretor de ACTH es el cateterismo bilateral de los senos petrosos inferiores, un método invasivo pero con una sensibilidad de $88 \%-100 \%$ y especificidad de $67 \%-100 \%{ }^{(2)}$. Este procedimiento involucra la cateterización de ambos senos petrosos inferiores, que constituyen el drenaje venoso proximal a la glándula hipofisaria. A partir del mismo se recolectan muestras para la medición de ACTH de ambos senos y de la periferia, en forma basal y luego del estímulo con hormona liberadora de adrenocorticotropina (CRH) o desmopresina. En Uruguay no hay publicaciones sobre la realización de esta técnica en el diagnóstico etiológico del síndrome de Cushing ACTH dependiente. El presente artículo tiene como objetivo presentar el primer caso en Uruguay en que se realizó la confirmación diagnóstica de EC mediante la realización del CSP.

\section{Caso clínico}

Mujer de 55 años, enviada de Colonia por endocrinóloga tratante, con antecedentes personales de obesidad, diabetes mellitus tipo 2, hipertensión y dislipemia. Como antecedentes ginecoobstétricos se destacan ciclos regulares previos, dos embarazos, dos cesáreas, y una histerectomía a los 50 años por miomatosis. Enviada para valoración por diagnóstico clínico presuntivo de SC, dado por historia de debilidad muscular proximal y equimosis fáciles, constatándose un "hábito cushingoide" al examen físico. Negaba consumo de glucocorticoides exógenos.

Se confirmó el diagnóstico bioquímico de hipercortisolismo mediante una prueba de supresión con dexametasona $1 \mathrm{mg}$ alterada y dos cortisoles libres urinarios ine- quívocamente elevados, ACTH dependiente por presentar dos dosificaciones de ACTH $>20 \mathrm{pg} / \mathrm{ml}$ (tabla 1). Para determinar la localización hipofisaria o ectópica, se solicitó una prueba de supresión fuerte con dexametasona 8 $\mathrm{mg}$, que orientó a un tumor hipofisario por cursar con una supresión del cortisol mayor al 50\% del valor basal (tabla 1). No contamos con disponibilidad de CRH en nuestro medio, por lo que no realizamos test de estímulo con dicha hormona. La RNM de cráneo con enfoque selar mostró una imagen sugestiva de adenoma hipofisario con un diámetro de $4 \mathrm{~mm}$ (figura 1). No contamos con fotos de RNM en T1 luego de la administración de gadolinio.

Por presentar un hipercortisolismo ACTH dependiente con una imagen adenohipofisaria compatible con un adenoma $<6 \mathrm{~mm}$, se decide realizar el CSP con estímulo con desmopresina para confirmar la localización de la hipersecreción de ACTH.

Las determinaciones de $\mathrm{ACTH}$, cortisol y prolactina se realizaron mediantes inmunoensayos inmunométricos en el analizador multiparamétrico Cobas 6000 de Roche en el Departamento de Laboratorio Clínico del Hospital de Clínicas.

Se confirmó el hipercortisolismo en la mañana del procedimiento mediante un cortisol hora $8>10 \mathrm{mcg} / \mathrm{dl}$. Se realizó punción venosa femoral bilateral y se colocaron introductores $6 \mathrm{~F}$. Se realizó flebografía, comprobando la permeabilidad de la vena yugular interna únicamente del lado derecho. Se ascendió un catéter Launcher $5 \mathrm{~F}$ MP y en forma coaxial un microcateter Echelon con microguía Excelsior SL 10 que se posicionó en el seno petroso inferior comprobando su posición bajo fluoroscopía. En las imágenes se aprecia en frente y perfil el microcatéter Echelon con sus marcas radioopacas posicionado en el seno petroso inferior derecho y se observa contraste en el mismo luego de una inyección retrógrada superselectiva en seno petroso para corroborar la posición (figura 2). No se pudo cateterizar el seno petroso izquierdo por razones anatómicas. Se tomaron muestras de sangre venosa del seno petroso cateterizado y de una vena periférica en forma basal (10 y 5 minutos previa administración de desmopresina) para medición de ACTH, cortisol y prolactina. Luego se administró una infusión de $10 \mu \mathrm{g}$ intravenosos de desmopresina y se tomaron nuevas muestras para iguales mediciones posteriores al estímulo $(3,5,10$ y 15 minutos después de la administración de desmopresina).

El gradiente petroso-periférico fue $>2$ en forma basal y $>3$ luego del estímulo con desmopresina, confirmando el diagnóstico de EC (tabla 2, figura 3). No hubo complicaciones durante el procedimiento. Se realizó la resección del adenoma mediante cirugía transesfenoidal, con buena evolución posterior. La inmunohistoquímica mostró tinción para $\mathrm{ACTH}$. 
Tabla 1. Valoración bioquímica.

\begin{tabular}{lccc}
\hline Bioquímica & Valor 1 & Valor 2 & Rango de referencia \\
\hline Cortisol post PSD 1 mg & 22,2 & - & $>1,8 \mu \mathrm{gg} / \mathrm{dl}$ \\
Cortisol libre urinario & 345,4 & 245 & $20-70 \mu \mathrm{gg} / 24 \mathrm{~h}$ \\
ACTH & 51,7 & 74,1 & Indetectable-46 pg/ml \\
Cortisol basal y post PSD 8 mg & 24,4 (basal) & 10 (post 8mg) & $\mu \mathrm{gg} / \mathrm{dl}$ \\
\hline
\end{tabular}

PSD 1 mg: Prueba de supresión con dexametasona 1 mg; PSD 8 mg: Prueba de supresión con dexametasona 8 mg.

Tabla 2. Resultado del CSP.

\begin{tabular}{cccccccc}
\hline & \multicolumn{3}{c}{ ACTH $(\mathrm{pg} / \mathrm{ml})$} & & PRL $(\mathrm{ng} / \mathrm{ml})$ & Cortisol $(\mu \mathrm{g} / \mathrm{dl})$ \\
\cline { 2 - 6 } & $S P D$ & $P$ & $\begin{array}{c}\text { Gradiente } A C T H \\
\text { SPD/P }\end{array}$ & SPD & $P$ & $\begin{array}{c}\text { Gradiente PRL } \\
\text { SPD/P }\end{array}$ \\
\hline-10 & 933 & 71 & 13 & 192 & 18 & 11 & 21 \\
-5 & $>2.000$ & 64 & 31 & $>470$ & 18 & 26 & 19 \\
10 & $>2.000$ & 265 & 8 & $>470$ & 17 & 28 & 26 \\
15 & $>2.000$ & 132 & 15 & 378 & 16 & 24 & 34 \\
20 & $>2.000$ & 52 & 38 & 442 & 16 & 28 & 36 \\
\hline
\end{tabular}

Se extrae sangre 10 y 5 minutos previo y 10, 15 y 20 minutos post administración de desmopresina $10 \mu \mathrm{g}$. SPD: seno petroso derecho; P: periferia; PRL: prolactina.

\section{Discusión}

No hay consenso actual respecto a la indicación del CSP. Mientras algunos lo recomiendan como un estudio de rutina en cualquier paciente con hipercortisolismo ACTH dependiente ${ }^{(3-5)}$, otros sugieren reservarlo para pacientes con evidencia clínica y bioquímica de SC y hallazgos en la RNM negativos o equívocos (como adenomas $\leq 6 \mathrm{~mm})^{(1,6)}$. Si la RNM muestra un adenoma $>6 \mathrm{~mm}$, se verifica el tumor por cirugía en un $75 \%-98 \%{ }^{(7)}$. En cambio, sólo se verifica el tumor por cirugía en $52 \%$ de los pacientes con imagen $<6 \mathrm{~mm}^{(8)}$. Además, la RNM no logra identificar imagen del tumor hipofisario en hasta el $40 \%$ de los pacientes con EC, y pueden hallarse incidentalomas hipofisarios en al menos un $10 \%$ de la población general ${ }^{(9)}$.

Mediante el CSP se demuestra la hipersecreción de ACTH a nivel hipofisario al documentar un gradiente de ACTH central a periférico en el drenaje del tumor (figura 4$)^{(10)}$. Se miden los niveles de ACTH en ambos senos petrosos y en una vena periférica, tanto en condiciones basales como tras estímulo con CRH. Dicho estímulo tiene como objetivo mejorar la sensibilidad del procedi-

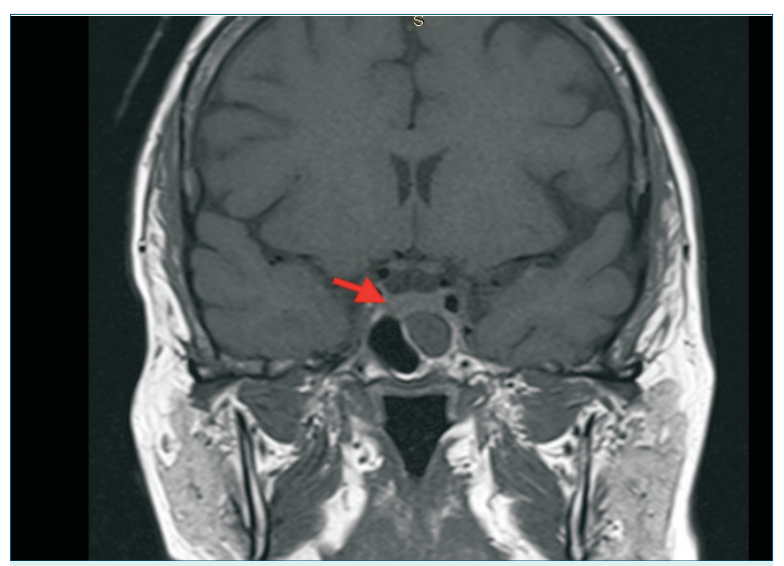

Figura 1. RNM de región selar, T1 sin contraste. Se evidencia pequeña zona de hipointensidad (flecha roja).

miento. El uso de desmopresina en lugar de $\mathrm{CRH}$ es una alternativa segura con resultados comparables ${ }^{(11-13)}$. El CSP tiene un alto rendimiento en el diagnóstico del SC en centros experimentados. Se ha reportado una sensibi- 

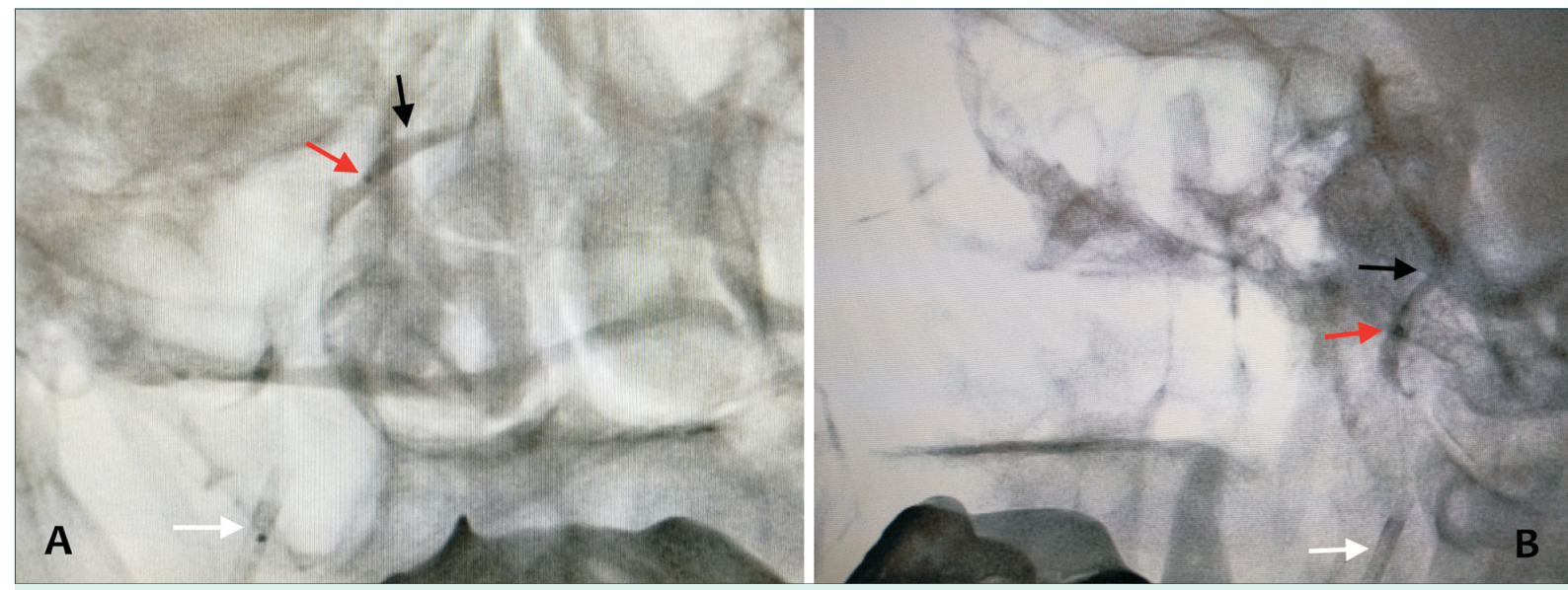

Figura 2. Venografía por fluroscopía. A) Frente. B) Perfil. Se evidencia el catéter guía (flecha blanca) y microcatéter Echelon con sus marcas radioopacas (flechas rojas) posicionado en el seno petroso inferior derecho. Se observa la inyección de contraste (flecha negra).

lidad entre $88 \%-100 \%$ y una especificidad entre $67 \%-100 \%{ }^{(2)}$. Un metaanálisis de 21 estudios reportó una sensibilidad de $96 \%$ y una especificidad de $100 \%$. La sensibilidad aumenta hasta el $100 \%$ con la estimulación con CRH o desmopresina ${ }^{(7)}$.

El procedimiento debe realizarse en presencia de hipercortisolemia, que suprime tanto la actividad secretora basal como estimulada de las células corticotróficas normales, de forma que la ACTH medida sea el resultado de la actividad secretora del tejido tumoral. Una relación entre las concentraciones de ACTH central y periférica $\geq 2$ en condiciones basales $\mathrm{o} \geq 3$ tras estímulo, indica un origen hipofisario de la secreción de $\mathrm{ACTH}^{(14)}$.

Dentro de los falsos negativos, se destacan la inadecuada cateterización o las alteraciones anatómicas, y dentro de los falsos positivos, la secreción cíclica de $\mathrm{ACTH}$ por un tumor ectópico que produciría una supresión incompleta de la ACTH hipofisaria ${ }^{(2)}$. Los falsos negativos son más frecuentes que los positivos, con una prevalencia reportada de entre $3 \%-19 \%{ }^{(15-17)}$.

Algunos estudios sugieren que la medición de hormonas de la hipófisis anterior puede ser útil para evitar falsos positivos. La utilidad de medir la prolactina es para documentar la cateterización exitosa (gradiente de prolactina en $\mathrm{SP} /$ periferia $\geq 1,8$ ). Asimismo, puede ayudar a diferenciar entre EC y tumor ectópico si no hay criterios en los gradientes de $\mathrm{ACTH}$, mediante la división de los gradientes de ACTH SP/periferia con los de PRL $\mathrm{SP} /$ periferia $\left(>1.3\right.$ consistente con EC) ${ }^{(18-20)}$.

El efecto adverso más comúnmente reportado del CSP es el hematoma en el sitio de acceso a la vena, que se ha reportado en $3 \%-4 \%$ de los pacientes ${ }^{(21)}$. La frecuencia de complicaciones severas es menor al 1\% cuando es realizado por radiólogos experimentados (ac-

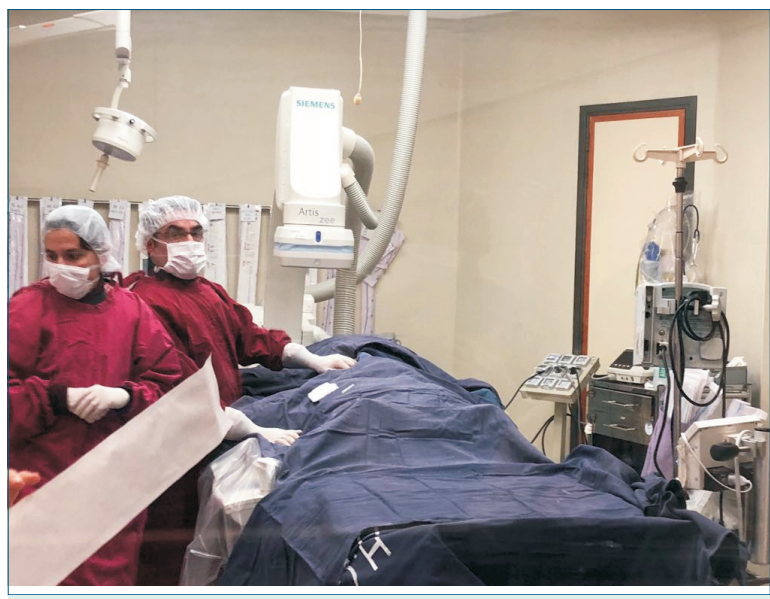

Figura 3. Durante el procedimiento del cateterismo de senos petrosos.

cidente cerebrovascular, tromboembolismo pulmonar, trombosis venosa profunda) $)^{(2)}$.

\section{Conclusiones}

La diferenciación entre las causas de hipercortisolismo ACTH dependiente es crucial para establecer un tratamiento adecuado para cada condición. El CSP es actualmente el método más preciso para demostrar hipersecreción hipofisaria de cortisol. Es un procedimiento invasivo, pero la aparición de eventos adversos es extremadamente rara cuando es realizado por radiólogos expertos.

Creemos que es un gran avance para el país disponer de este procedimiento, que es el patrón de oro para diferenciar EC de tumor ectópico secretor de $\mathrm{ACTH}$, en casos en los que los exámenes no son concluyentes. 

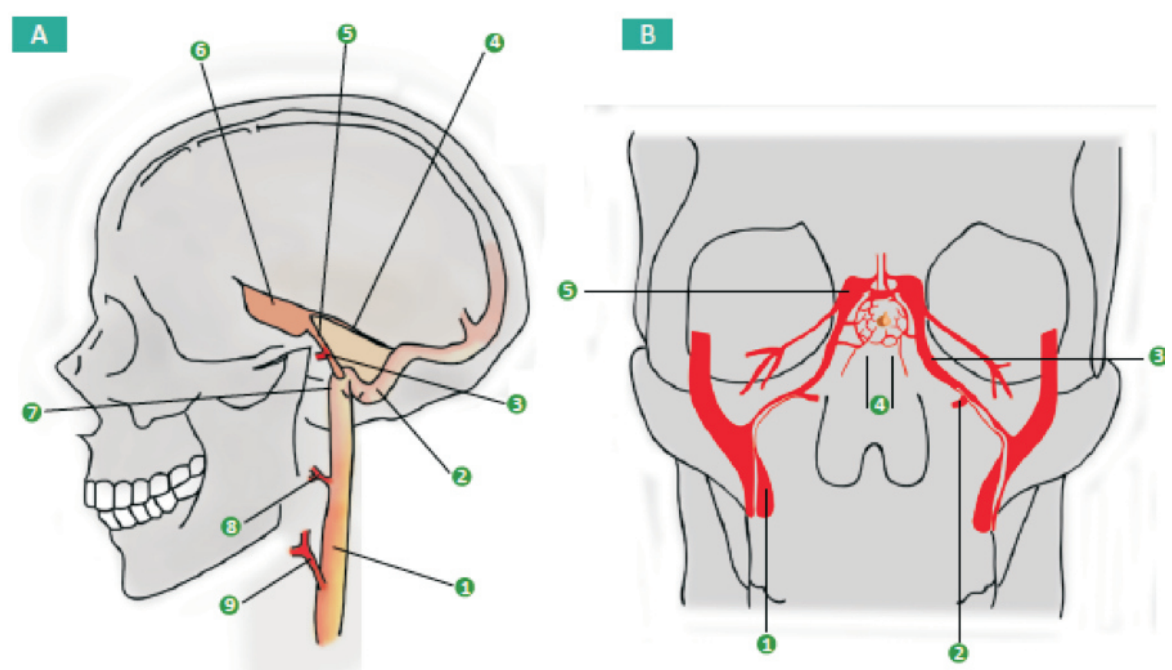

Figura 4. Drenaje venoso de la hipófisis. Los senos cavernosos drenan por los senos petrosos inferiores, que a su vez desembocan en las venas yugulares internas a través de los senos sigmoides. A) Corte sagital. (1) Vena yugular interna. (2) Seno sigmoideo. (3) Vena condilar anterior. (4) Seno petroso superior. (5) Seno petroso inferior (6) Seno cavernoso. (7) Bulbo yugular. (8) Vena faríngea. (9) Vena facial. B) Corte coronal. (1) Vena yugular. (2) Vena condilaranterior. (3) Seno petroso inferior. (4) Venas hipofisarias confluentes. (5) Senos cavernosos. Reproducido con autorización de Montoya y col ${ }^{(10)}$.

\section{Summary}

Identifying the cause of adrenocorticotropin (ACTH)-dependent Cushing's syndrome is key to define the appropriate treatment. Hypersecretion of the adrenocorticotropic hormone (ACTH) is mainly caused by a pituitary tumor (Cushing's syndrome) or, in $10 \%$ to $20 \%$ of cases, by tumors with ectopic production of this hormone. Differentiation between these two etiologies may not be easy due to the low sensitivity and specificity of noninvasive tests. Bilateral sampling of the lower petrosal sinus is the gold standard to differentiate between a pituitary and an ectopic origin, showing the pituitary ACHT hypersecretion and recording the central-to-peripheral ACTH gradient in the tumor's drainage.

Despite it being highly recommended for all cases of ACTH-dependent Cushing's syndrome, it is reserved for patients with a diagnosis of hypercortisolism and negative or misleading findings in the MRI of the sellar region. The study presents the first case of petrosal sinus sampling for diagnostic purposes in Uruguay, in a 55-year-old woman with ACHT-dependent hypercortisolism showing an adenohypophysis image $<6 \mathrm{~mm}$. The petrosal-peripheral gradient confirmed the diagnosis of Cushing's syndrome and no complications arose during the procedure. Afterwards a transsphenoidal surgery was performed for resection of the adenoma. Evolution was good and immunochemistry confirmed the tumor's etiology.

\section{Resumo}

Identificar a causa da síndrome de Cushing dependente de adrenocorticotropina (ACTH) é essencial para o tratamento adequado. A hipersecreção de ACTH se deve principalmente a um tumor hipofisário (doença de Cushing) ou, em 10\%-20\%, a tumores com produção ectópica desse hormônio. Os testes não invasivos apresentam baixa sensibilidade e especificidade para diferenciar essas duas etiologias. O padrão ouro é o cateterismo bilateral dos seios petrosos inferiores (CEP). O CSP demonstra hipersecreção de ACTH no nível da hipófise, documentando um gradiente de ACTH central a periférico na drenagem do tumor. É recomendado nos casos de síndrome de Cushing dependente de ACTH, embora seja geralmente reservado para pacientes com diagnóstico de hipercortisolismo e achados negativos ou duvidosos na ressonância magnética (RNM) da região selar.

Apresentamos o primeiro caso no Uruguai em que o CSP foi usado como método diagnóstico, uma mulher de 55 anos que apresentava hipercortisolismo ACTH dependente com imagem da hipófise anterior $<6 \mathrm{~mm}$. O gradiente petroso-periférico confirmou o diagnóstico de doença de Cushing e não houve complicações durante o procedimento. A seguir, o adenoma foi ressecado por cirurgia transesfenoidal, com boa evolução e confirmação imunohistoquímica do tumor. 


\section{Bibliografía}

1. Arnaldi G, Angeli A, Atkinson AB, Bertagna X, Cavagnini F, Chrousos GP, et al. Diagnosis and complications of Cushing's syndrome: a consensus statement. J Clin Endocrinol Metab 2003; 88(12):5593-602.

2. Zampetti B, Grossrubatscher E, Ciaramella PD, Boccardi E, Loli P. Bilateral inferior petrosal sinus sampling. Endocr Connect 2016; 5(4):R12-25.

3. McCance DR, McIlrath E, McNeill A, Gordon D, Hadden D, Kennedy L, et al. Bilateral inferior petrosal sinus sampling as a routine procedure in ACTH-dependent Cushing's syndrome. Clin Endocrinol (Oxf) 1989; 30(2):157-66.

4. Findling JW, Kehoe ME, Shaker JL, Raff H. Routine inferior petrosal sinus sampling in the differential diagnosis of adrenocorticotropin (ACTH)-dependent Cushing's syndrome: early recognition of the occult ectopic ACTH syndrome. J Clin Endocrinol Metab 1991; 73(2):408-13.

5. Newell-Price J. Diagnosis/differential diagnosis of Cushing's syndrome: a review of best practice. Best Pract Res Clin Endocrinol Metab 2009; 23(Suppl 1):S5-14.

6. Findling JW, Raff H. Cushing's syndrome: important issues in diagnosis and management. J Clin Endocrinol Metab 2006; 91(10):3746-53 .

7. Newell-Price J, Trainer P, Besser M, Grossman A. The diagnosis and differential diagnosis of Cushing's syndrome and pseudo-Cushing's states. Endocr Rev 1998; 19(5):64772.

8. Yogi-Morren D, Habra MA, Faiman C, Bena J, Hatipoglu B, Kennedy L, et al. Pituitary MRI findings in patients with pituitary and ectopic acth-dependent Cushing syndrome: does a 6-mm pituitary tumor size cut-off value exclude ectopic acth syndrome? Endocr Pract 2015; 21(10): 1098-103.

9. Salenave S, Gatta B, Pecheur S, San-Galli F, Visot A, Lasjaunias $\mathbf{P}$, et al. Pituitary magnetic resonance imaging findings do not influence surgical outcome in adrenocorticotropin-secreting microadenomas. J Clin Endocrinol Metab 2004; 89(7):3371-6.

10. Montoya J, Builes C, Gutiérrez J, Campuzano G. Muestreo de senos petrosos inferiores en el diagnóstico de pacientes con síndrome de Cushing dependiente de hormona adrenocorticotrópica. Med Lab 2013; 19(9-10):411-50.

11. Deipolyi AR, Hirsch JA, Oklu R. Bilateral inferior petrosal sinus sampling with desmopressin. J Neurointerv Surg 2013; 5(5):487-8.
12. Castinetti F, Morange I, Dufour H, Jaquet P, Conte-Devolx B, Girard N, et al. Desmopressin test during petrosal sinus sampling: a valuable tool to discriminate pituitary or ectopic ACTH-dependent Cushing's syndrome. Eur J Endocrinol 2007; 157(3):271-7.

13. Machado MC, De Sa SV, Domenice S, Fragoso MCBV, Puglia P, Pereira MAA, et al. The role of desmopressin in bilateral and simultaneous inferior petrosal sinus sampling for differential diagnosis of ACTH-dependent Cushing's syndrome. Clin Endocrinol (Oxf) 2007; 66(1):136-42.

14. Oldfield EH, Doppman JL, Nieman LK, Chrousos GP, Miller DL, Katz DA, et al. Petrosal sinus sampling with and without corticotropin-releasing hormone for the differential diagnosis of Cushing's syndrome. N Engl J Med 1991; 325(13):897-905.

15. Sheth SA, Mian MK, Neal J, Tritos NA, Nachtigall L, Klibanski A, et al. Transsphenoidal surgery for cushing disease after nondiagnostic inferior petrosal sinus sampling. Neurosurgery 2012; 71(1):14-22.

16. López J, Barceló B, Lucas T, Salame F, Alameda C, Boronat $\mathbf{M}$, et al. Petrosal sinus sampling for diagnosis of Cushing's disease: evidence of false negative results. Clin Endocrinol (Oxf) 1996; 45(2):147-56.

17. Doppman JL, Chang R, Oldfield EH, Chrousos G, Stratakis CA, Nieman LK. The hypoplastic inferior petrosal sinus: a potential source of false-negative results in petrosal sampling for Cushing's disease. J Clin Endocrinol Metab 1999; 84(2):533-40.

18. Mulligan GB, Eray E, Faiman C, Gupta M, Pineyro MM, Makdissi A, et al. Reduction of false-negative results in inferior petrosal sinus sampling with simultaneous prolactin and corticotropin measurement. Endocr Pract 2011; 17(1):33-40.

19. Sharma ST, Raff H, Nieman LK. Prolactin as a marker of successful catheterization during IPSS in patients with ACTH-dependent Cushing's syndrome. J Clin Endocrinol Metab 2011; 96(12):3687-94.

20. Mulligan GB, Faiman C, Gupta M, Kennedy L, Hatipoglu B, Hui F, et al. Prolactin measurement during inferior petrosal sinus sampling improves the localization of pituitary adenomas in Cushing's disease. Clin Endocrinol (Oxf) 2012; 77(2):268-74.

21. Deipolyi A, Karaosmanoglu A, Habito C, Brannan S, Wicky S, Hirsch J, et al. The role of bilateral inferior petrosal sinus sampling in the diagnostic evaluation of Cushing syndrome. Diagn Interv Radiol 2012; 18(1):132-8. 


\section{Contribución de autores}

Mariana Pintado, https://orcid.org/0000-0003-3202-5328. Concepción, diseño, ejecución, análisis, interpretación de los resultados, redacción, revisión crítica.

Paula Montiglia, https://orcid.org/0000-0001-7030-6038. Concepción, diseño, ejecución, análisis, interpretación de los resultados, revisión crítica.

Marcelo Langleib, https://orcid.org/0000-0003-1827-0765. Concepción, diseño, ejecución, análisis, interpretación de los resultados, revisión crítica.

Fernanda Sánchez, https://orcid.org/0000-0002-0774-8607. Concepción, diseño, ejecución, análisis, interpretación de los resultados, revisión crítica.

Flavia Varela, https://orcid.org/0000-0001-6204-7910. Concepción, diseño, ejecución, análisis, interpretación de los resultados, revisión crítica. Ramiro Lima, https://orcid.org/0000-0003-1697-5163. Concepción, diseño, ejecución, análisis, interpretación de los resultados, revisión crítica. Margarita Vaz, https://orcid.org/0000-0002-3805-1191. Concepción, diseño, ejecución, análisis, interpretación de los resultados, revisión crítica. Maria Mercedes Piñeyro, https://orcid.org/0000-0003-2083-7839. Concepción, diseño, ejecución, análisis, interpretación de los resultados, redacción, revisión crítica. 